\title{
TIME TRANSFER BY LASER LINK - THE T2L2 EXPERIMENT ON JASON-2 AND FURTHER EXPERIMENTS
}

\author{
ÉTIENNE SAMAIN*, JONATHAN WEICK, PATRICK VRANCKEN, FRANCK PARA, \\ DOMINIQUE ALBANESE, JOCELYN PARIS and JEAN-MARIE TORRE \\ Observatoire de la Côte d'Azur, UMR Gemini, R\&D Métrologie, \\ 06460 Caussols, France \\ *etienne.samain@obs-azur.fr \\ CHENG ZHAO \\ Purple Mountain Observatory, Chinese Academia of Sciences, Nanjing 210008, China \\ PHILIPPE GUILLEMOT and ISABELLE PETITBON \\ Centre National d'Études Spatiales, 31400 Toulouse, France
}

Received 9 October 2006

Revised 21 February 2007

Communicated by W.-T. Ni

\begin{abstract}
The new generation of optical time transfer will allow the synchronization of remote ultra stable clocks and the determination of their performances over intercontinental distances. The principle of T2L2 (Time Transfer by Laser Link) is based on the techniques of satellite laser ranging coupled with time-frequency metrology. It consists of synchronizing ground and space clocks using short laser pulses travelling between ground clocks and satellite equipment. The instrument will be integrated on the ocean altimetry satellite Jason-2 that is scheduled for launch in 2008. ${ }^{\mathrm{a}}$ The experiment should enhance the performance of time transfer by one or two magnitudes compared to existing microwave techniques such as GPS and Two-Way Satellite Time and Frequency Transfer (TWSTFT).
\end{abstract}

Keywords: Laser ranging; time-frequency; synchronization.

\section{Introduction}

The Time Transfer by Laser Link experiment (T2L2), ${ }^{1-5}$ under development at OCA (Observatoire de la Côte d'Azur) and CNES (Centre National d'Études Spatiales), France, will be launched in 2008 on the altimetric satellite Jason-2. ${ }^{a}$ The experiment principle is issued from satellite laser ranging, i.e. the timing of transmitted and reflected laser pulses. T2L2 on Jason-2 will make it possible to synchronize remote ground clocks and compare their frequency stabilities with a

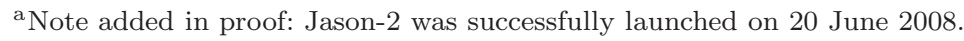


performance never reached before. T2L2 will allow synchronization of a ground and space clock and measurement of the stability of remote ground clocks over continental distances, itself having a time stability in the range of $1 \mathrm{ps}$ over $1000 \mathrm{~s}$.

This paper will shortly explain the T2L2 optical time transfer principle and give an overview of the instrumental heritage up to the recent adoption on Jason-2. After a description of the Jason-2 mission, more details will be given concerning the T2L2 instrument, the stakes and the objectives of the project, supplemented by a preliminary performance budget based on measurements conducted by OCA through a ground experiment performed in 2004 and several tests on electronic prototypes. Finally, some possibilities of further projects ${ }^{6-11}$ linked to the T2L2 experiment or utilizing related technologies will be described.

\section{The T2L2 Experiment}

\subsection{Principle of T2L2}

T2L2 allows the synchronization of remote clocks on Earth and the monitoring of satellite clocks. The principle is based on the propagation of light pulses between laser stations and a satellite equipped with specific instrumentation. The role of the space segment is to work as a sort of relay between the different ground clocks that are to be synchronized. The T2L2 payload comprises a photodetection device and a time-tagging unit. ${ }^{12}$ The experiment also uses the Jason-2 laser ranging array (LRA) and the Doris ultra-stable oscillator USO. The laser station emits short asynchronous laser pulses towards the satellite and the LRA corner-cubes return a fraction of the received photons back to the station (Fig. 1). The station records the start $\left(t_{\text {start }}\right)$ and return $\left(t_{\text {return }}\right)$ time of each light pulse. The T2L2 payload records the arrival time $\left(t_{\text {board }}\right)$ in the temporal reference frame of the onboard oscillator. These data are regularly downloaded to the ground via a regular microwave communication link.
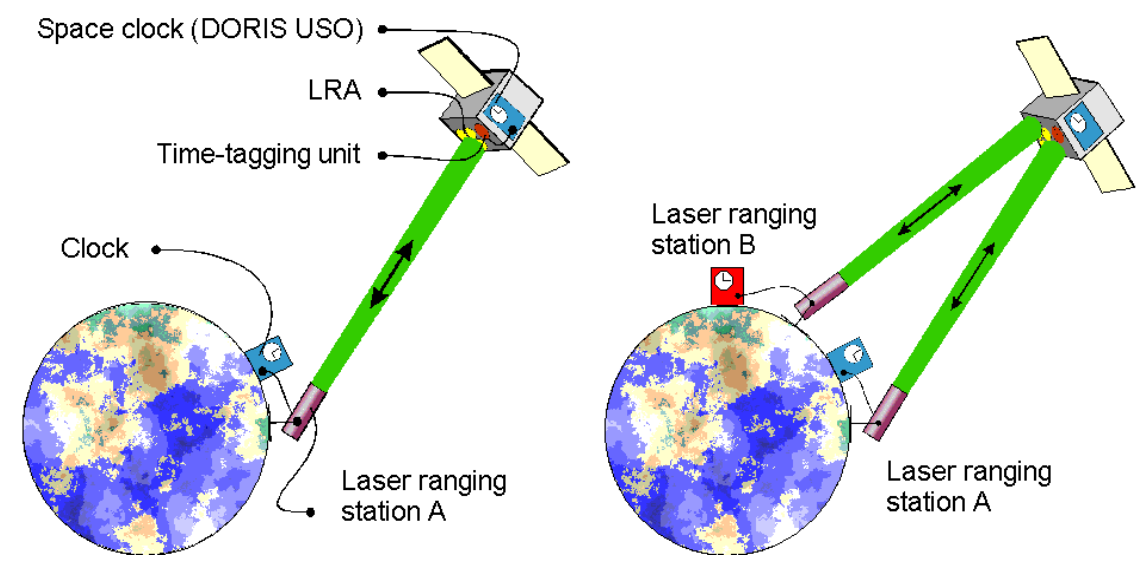

Fig. 1. The T2L2 experiment principle: single and common view pass. 
For a given light pulse emitted from a ground station, the time offset $\chi_{A S}$ between ground clock $\mathrm{A}$ and space clock $\mathrm{S}$ is deduced from the measurement triplets $\left\{t_{\text {start }}, t_{\text {board }}, t_{\text {return }}\right\}$ with the following time equation:

$$
\chi_{A S}=\frac{t_{\text {start }}+t_{\text {return }}}{2}-t_{\text {board }}+\tau_{\text {relativity }}+\tau_{\text {atmosphere }}+\tau_{\text {geometry }}
$$

- $t_{\text {start }}$ is the departure date of the light pulse from the ground station,

- $t_{\text {return }}$ is the return date of the light pulse reflected by LRA,

- $t_{\text {board }}$ is the detection date of the light pulse at the instrument,

- $\tau_{\text {relativity }}$ considers relativistic effects,

- $\tau_{\text {atmosphere }}$ is a correction term for atmospheric effects,

- $\tau_{\text {geometry }}$ takes account of the geometrical offset between the reflection and detection equivalent points, further depending on elevation and attitude of the satellite.

In common view configuration, with two laser ranging stations $\mathrm{A}$ and $\mathrm{B}$ firing towards the satellite simultaneously, the noise of the on-board oscillator has to be considered over very short time, in such a way that it can be considered negligible in the global error budget. The time transfer between ground clocks A and B is then deduced from the difference between $\chi_{A S}$ and $\chi_{B S}$. In a non-common view mode, the temporal information is carried by the satellite local oscillator over the distance separating the two ground stations visibility. The respective time walk of the on-board clock has then to be taken into account.

\subsection{Historical account}

The concept of transferring time using optical devices was born in 1972 upon an ESA (European Space Agency) and CNES initiative under the name LASSO (LAser Synchronization from Stationary Orbit). A first optical time transfer had been successfully achieved in 1992 between OCA, France and McDonald Observatory, Texas with LASSO on the Meteosat P2 geostationary satellite. This experiment was able to measure a stability of $10^{-13}$ over $1000 \mathrm{~s}$, validating the feasibility of the concept.

T2L2 was first proposed in 1996 to fly on the Russian space station Mir taking the opportunity of the French PERSEUS mission, but the project was finally stopped at the end of the phase A. In the meantime it was accepted by ESA in the framework of the ACES (Atomic Clock Ensemble in Space) program scheduled on the International Space Station (ISS). T2L2 was one of the three scientific proposals of ACES, but had to be descoped in 2001 for some reasons concerning the whole ACES mission. Feasibility studies were led by CNES for other flight opportunities (Myriade Micro-satellite, Galileo Test Bed), ${ }^{13}$ but finally a new opportunity appeared at the end of 2004, when NASA decided to abandon the WSOA instrument, an American contribution to the Jason-2 mission. A preliminary analysis conducted at CNES confirmed the high interest in putting the T2L2 instrument onto this altimetry-dedicated space vehicle and led CNES to select the T2L2 instrument as a passenger on the Jason-2 mission. 


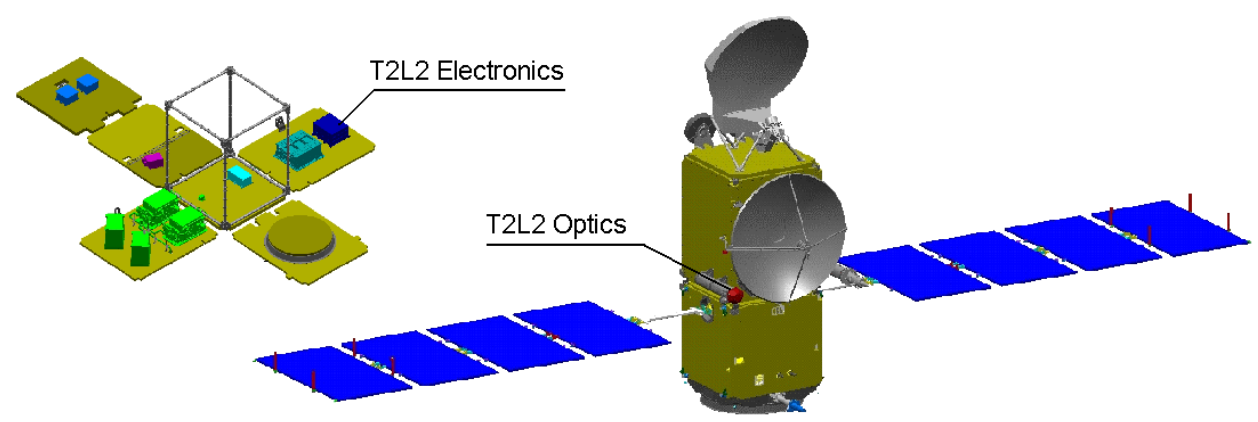

Fig. 2. The integration of the T2L2 device inside the Jason-2 spacecraft.

\subsection{T2L2 on Jason-2}

Jason-2 is a French-American follow-on mission to Jason-1 and Topex/Poseidon. Conducted by NASA and CNES, its goal is to study the internal structure and dynamics of ocean currents mainly by radar altimetry. The Jason-2 satellite is based on the French recurrent Proteus platform (Fig. 2) and equipped with the dual-frequency radar altimeter Poseidon-3 and a microwave radiometer to measure the water vapor contained in the troposphere and derive the appropriate radar path delay correction. For the needs of precise determination of the satellite orbit, three independent positioning systems are also included: a Doris transponder, a GPS receiver and the LRA (Laser Ranging Array) retroreflector, an ensemble of corner cubes for precise satellite laser ranging. The T2L2 instrument and two radiation studying payloads (Carmen-2, France and LPT, Japan) are additional passenger payloads that were accepted on Jason 2 in order to supplement the primary mission.

The satellite will be placed in a $1,336 \mathrm{~km}$ orbit with $66^{\circ}$ inclination by a Delta launcher. This orbit allows common views at continental scale (about $3000 \mathrm{~km}$ baseline between stations). The time interval between two passes varies from 2 to 14 hours with an average duration of about $1000 \mathrm{~s}$.

The T2L2 specific instrumentation has an estimated mass of $10 \mathrm{~kg}$ and a power consumption of $42 \mathrm{~W}$. It is composed of an optical and an electronic subsystem (Fig. 3):

- Two photo detection units are located outside the main Jason-2 payload on the LRA boom. Both are composed of avalanche photodetectors. The first one is working in a special non-linear "Geiger" mode for precise chronometry. The other works in linear gain mode in order to trigger the whole detection chain and to measure the received optical energy and the continuous noise flux originating in the earth albedo. To minimize the false detection rate, the detection threshold may be adjusted either by remote control or automatically as a function of the continuous flux measurement. 
- The electronic unit, located inside the Jason-2 payload module is composed of two main items. The detection unit ensures the conversion of the laser pulse into an electronic signal and the time-tagging unit ${ }^{12}$ proceeds to its timing. The unit also comprises the control electronics for the whole instrument.

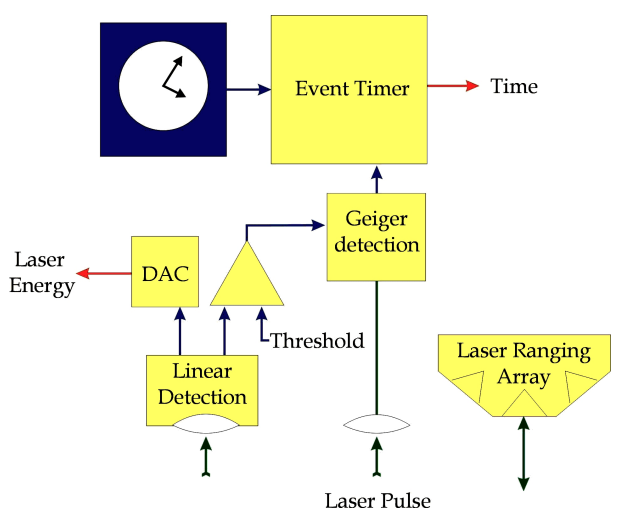

Fig. 3. Synoptic of the T2L2 space instrument.

\subsection{Mission's objectives}

The objectives of the T2L2 experiment on Jason-2 are threefold:

- Validation of optical time transfer, including the validation of the experiment, its time stability and accuracy. T2L2 will be a first step and a demonstration for future experiments based on one way laser ranging ${ }^{11}$ techniques: TIPO. It should further allow the decorrelation of the effects coming from the retroreflector signature. In that way, it will permit to improve the precision of the telemetry.

- Scientific applications concerning time and frequency metrology allowing the calibration of radiofrequency time transfer (GPS and Two-Way), fundamental physics with the measurement of light speed anisotropy and alpha fine structure constant, Earth observation and very long baseline interferometry (VLBI).

- Characterization of the on-board Doris oscillator's drift, especially above the South Atlantic Anomaly (SAA). The two radiation instruments aboard will give the possibility to find a correlation between the expected and measured drift and propose adequate corrections.

\subsection{Preliminary performance budget}

\subsubsection{T2L2 ground experiment}

A ground-to-ground time transfer experiment has been led at OCA in 2004 to assess the whole error budget. ${ }^{14,15}$ A retro-reflector was placed on a near mountain (2.5 
$\mathrm{km}$ ), whereas the space segment was installed in front of the telescope: The laser beam was thus bent two times, travelling from the ground equipment (i.e. the laser station) to the space segment, and the same way back. This close-by configuration between the ground and space segment allowed the comparison between the clock's phase drift obtained through the T2L2 experiment and the phase measured directly. Stability measurements, noise estimations and dedicated sensitivity measurements could be deduced: the short-term white phase noise $\sigma_{x}(\tau)=17 \cdot 10^{-12} \times \tau^{-\frac{1}{2}}$ $@ \tau_{0}=1 \mathrm{~s}$ and the long-term drift of about $5 \mathrm{ps} /$ day are very close to the time stability obtained within a common clock experiment.

\subsubsection{Ground to satellite time transfer}

The time stability for the time transfer between a ground clock A and the on-board clock $\mathrm{S}$ is derived from Eq. (1) to:

$$
\sigma_{x, A S}^{2}(\tau)=\frac{\sigma_{x, s}^{2}(\tau)}{4}+\frac{\sigma_{x, r}^{2}(\tau)}{4}+\sigma_{x, b}^{2}(\tau)+\sigma_{x, \text { Geom }}^{2}(\tau)+\sigma_{x, A t m}^{2}(\tau)+\sigma_{x, \text { Rel }}^{2}(\tau) .
$$

$\sigma_{x, s}$ is the timing stability for the start laser pulse and is a combination of the uncertainties coming from the detector and the time-tagging. The detector has three noise sources: an intrinsic noise corresponding to the random multiplication of electron-holes pairs inside the cavity, a noise which is depending on the energetic flux and a noise which is function of the laser pulse width. The time-tagging noises are linked to its non-linearity behavior, its intrinsic noise in a synchronous mode and its calibration's defects. The stability generally is:

$$
\sigma_{x, s}^{2}(\tau)=\left(4 \cdot 10^{-12} \times \tau^{-\frac{1}{2}}\right)^{2}+\left(10 \cdot 10^{-15} \times \tau^{\frac{1}{2}}\right)^{2} .
$$

$\sigma_{x, r}$ is the timing stability for the return laser pulse and is highly linked to the instrumentation. Considering a detection device based on an avalanche photodiode working in Geiger mode coupled with a linear detector to evaluate the photon flux:

$$
\sigma_{x, r}^{2}(\tau)=\left(30 \cdot 10^{-12} \times \tau^{-\frac{1}{2}}\right)^{2}+\left(10 \cdot 10^{-15} \times \tau^{\frac{1}{2}}\right)^{2} .
$$

$\sigma_{x, b}$ is the timing stability of the embarked instrument. The detector and timetagging stabilities are of the same kind as those described for the return date. Special considerations have to be made because of the independence of the two channels: evolution of the transmissivity ratio with the parameters attitude, energy, temperature, ageing etc. and also photon noise at low flux level. The on-board stability of the instrument can be written as:

$$
\sigma_{x, b}^{2}(\tau)=\left(12.6 \cdot 10^{-12} \times \tau^{-\frac{1}{2}}\right)^{2}+\left(12.6 \cdot 10^{-15} \times \tau^{\frac{1}{2}}\right)^{2} .
$$

$\sigma_{x, \text { Geom }}$ is the geometric noise due to the fact that the reference point of detection is dissociated from the reflection point. Some laboratory experiments were made with the French Transportable Laser Ranging Station (FTLRS) in order to evaluate this parameter:

$$
\sigma_{x, \text { Geom }}^{2}(\tau)=\left(10 \cdot 10^{-12} \times \tau^{-\frac{1}{2}}\right)^{2} .
$$




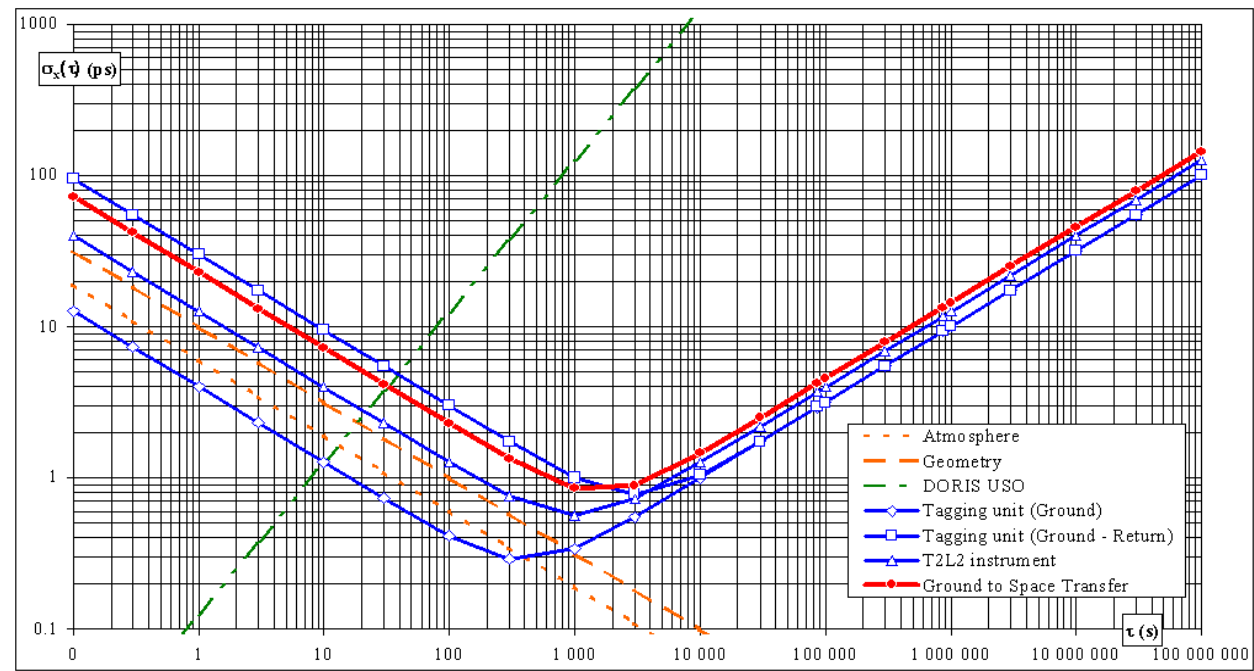

Fig. 4. T2L2 ground to space time stability (expressed in $\sqrt{\mathrm{TVar}}$ ).

$\sigma_{x, A t m}$ considers the asymmetry in the atmospheric delay between the outward and return path of the laser pulse,

$$
\sigma_{x, A t m}^{2}(\tau)=\left(6 \cdot 10^{-12} \times \tau^{-\frac{1}{2}}\right)^{2} \text { for } 0.1<\tau<10^{6} \mathrm{~s} .
$$

$\sigma_{x, R e l}$ represents the imprecision of the relativistic effects correction, what may be neglected. We obtain from Eq. (2) the time stability for the time transfer between a ground clock $\mathrm{A}$ and the on-board clock S (Fig. 4):

$$
\sigma_{A S}^{2}(\tau)=\left(23 \cdot 10^{-12} \times \tau^{-\frac{1}{2}}\right)^{2}+\left(14 \cdot 10^{-15} \times \tau^{\frac{1}{2}}\right)^{2} .
$$

\subsubsection{Ground to ground time transfer in common view}

For ground to ground clock comparisons, one has to consider the time transfer between the ground clock A and B. In a common view configuration, the dead time between the respective laser pulses can be considered negligible and we obtain:

$$
\sigma_{A B}^{2}(\tau)=\sigma_{A S}^{2}(\tau)+\sigma_{B S}^{2}(\tau)=\left(32 \cdot 10^{-12} \times \tau^{-\frac{1}{2}}\right)^{2}+\left(20 \cdot 10^{-15} \times \tau^{\frac{1}{2}}\right)^{2}
$$

In common view configuration, T2L2 should reach the majority of current atomic clocks (including cold atoms) for integration times exceeding $1000 \mathrm{~s}$.

\subsubsection{Ground to ground time transfer in non-common view}

In non-common view configuration, the noise of the Doris oscillator has to be taken into account. The time transfer stability depends on the time interval $\Delta \tau_{A B}$ between 


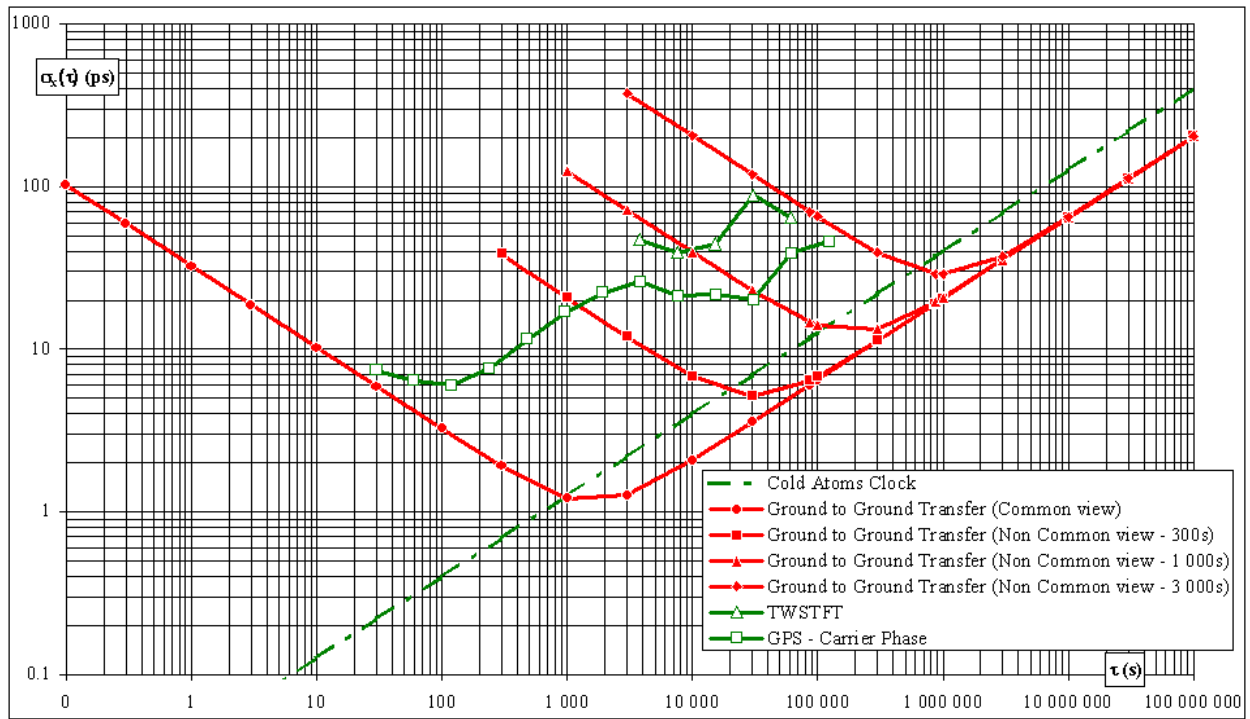

Fig. 5. T2L2 ground-to-ground time stability (expressed in $\sqrt{\text { TVar}}$ ) in common view and noncommon view configuration with various dead times.

access of each ground station. For different values of $\Delta \tau_{A B}$ (Fig. 5):

$$
\begin{aligned}
& \sigma_{x, A B, 300 s}^{2}(\tau)=\left(0.6 \cdot 10^{-9} \times \tau^{-\frac{1}{2}}\right)^{2}+\left(20 \cdot 10^{-15} \times \tau^{\frac{1}{2}}\right)^{2} . \\
& \sigma_{x, A B, 1000 s}^{2}(\tau)=\left(4 \cdot 10^{-9} \times \tau^{-\frac{1}{2}}\right)^{2}+\left(20 \cdot 10^{-15} \times \tau^{\frac{1}{2}}\right)^{2} . \\
& \sigma_{x, A B, 3000 s}^{2}(\tau)=\left(21 \cdot 10^{-9} \times \tau^{-\frac{1}{2}}\right)^{2}+\left(20 \cdot 10^{-15} \times \tau^{\frac{1}{2}}\right)^{2} .
\end{aligned}
$$

In non-common view, with the limitation imposed by the on-board clock, T2L2 will still offer an interesting alternative in calibration campaigns of radiofrequency techniques as well as time and frequency transfer systems based on transportable stations.

\subsubsection{Discussion of the time transfer accuracy}

The above developed stability of the T2L2 experiment and the overall accuracy have to be considered separately. Overall accuracy characterises the capability of performing an absolute calibration of the ground and space equipment in order to allow a time scale comparison. The time transfer accuracy is in fact dominated by our capability to calibrate the equipment and to monitor their drifts. Calibration accuracy is difficult to evaluate, but a fine analysis of the ground and space equipment should lead to a level of below 100 ps. This order of performance represents an enhancement of one magnitude in accuracy as compared to the existing microwave links which are at the nanosecond level (see Table 1).

Reference clocks usually provide two output signals, one is sinusoidal at $10 \mathrm{MHz}$ or $5 \mathrm{MHz}$, the other is a PPS (one Pulse Per Second). Time scale comparisons are 
Table 1. Time transfer accuracy and stability for different techniques.

\begin{tabular}{lccc}
\hline Technique & Accuracy & $\begin{array}{c}\text { Stability over } 1000 \mathrm{~s} \\
\sqrt{\text { TVar }} \\
(\mathrm{ps})\end{array}$ & $\begin{array}{c}\text { Stability over 10 days } \\
\sqrt{\text { TVar }} \\
(\mathrm{ps})\end{array}$ \\
\hline GPS & few ns & 15 & $100-1000$ \\
TWSTFT & few ns & 15 & $100-1000$ \\
T2L2 & $<100 \mathrm{ps}$ & 1.2 & 6 \\
\hline
\end{tabular}

nowadays performed via the PPS signal. The uncertainty introduced by the measurement of the delay between the reference frequency used for timing and this PPS is estimated to be between 100 and 300 ps, mainly caused by the inhomogeneous propagation of the PPS signal into the cables. With the improvement of time transfer offered by T2L2, it becomes the most important source of uncertainty and the use of the sinusoidal signal will become necessary to avoid this kind of limitation.

\subsection{Current status}

The decision to put the T2L2 instrument in the Jason-2 satellite was taken in July 2005. The phase B started September 2005 and entered in phase C/D in January 2006. For the optics, only a proto-flight model has been envisioned, while the electronics are developed in three steps: prototype boards of the metrological part, engineering model (EM) and flight model (FM). Metrological tests on the EM have been performed in July 2006 and permitted to precisely evaluate the T2L2 performances. The measured time stability performance is close to the performance specifications that were deduced from a global performance budget. Tests on the FM are to be done in December for a delivery to Alcatel Space expected on January 2007. The results of the ground experiment performed in 2004 permitted to precisely evaluate the T2L2 performances. The tests that were so far conducted on the T2L2 electronics breadboard show the compliance with the metrology specifications for both the photo detection and the event timer. The Jason-2 launch is scheduled mid-2008 for a mission duration of three years (five years expected). The nominal T2L2 lifetime is two years.

\section{Further Projects}

\subsection{One way laser ranging TIPO}

As compared with T2L2, the major difference of TIPO ${ }^{11}$ (Télémétrie InterPlanétaire Optique) is the absence of a laser pulse reflection and thus a return date. The principle of the experiment relies on the derivation of the propagation delay of laser pulses emitted from Earth towards the spacecraft given by the difference between start and arrival time measured in the respective time scales of an on-board clock and the ground clock. The behaviour of the clocks is a major factor in the performance of the experiment. Even with compact clocks like Rubidium, ${ }^{13}$ having a 
good time stability over a few thousands of seconds, some interesting measurements can be realised. The TIPO scheme should be able to measure distances in the solar system up to a few billions of kilometres by this means. Further, the instrument should be able to measure short term distance variations very precisely (planetary gravity field, Shapiro signature, etc.). The data corresponding to the arrival times on the spacecraft are transmitted to the Earth via a classical microwave link. With a time stability of $\sigma_{x}=2.6 \cdot 10^{-13} \times \tau^{-\frac{1}{2}}$ one obtains a distance uncertainty on the centimeter level over an integration time of one day. Observations with several laser stations on Earth enable the measurement of the angular position of the spacecraft. This measurement requires a ground time transfer like T2L2 between participating laser stations in order to be able to precisely know the time intervals between laser pulses emitted by the Earth stations. The differential measurements of the arrival time on-board are processed in order to resolve the geometry of the system and deduce the angular position, i.e. the position of the spacecraft in the plan perpendicular to the line of sight. Since the measurement at the spacecraft level is differential, no long-term stability is required here. With distances between ground stations in the range of $10,000 \mathrm{~km}$, an uncertainty on the localisation of the station at the centimetre level and a time synchronisation between the stations of $30 \mathrm{ps}$, we get an uncertainty on the angular determination of $2 \times 10^{-9} \mathrm{rad}$. The signal detected on the spacecraft is given by:

$$
N_{L, e}=\frac{E \lambda}{h c} \frac{4}{\pi \theta^{2} d^{2}} \cdot \rho_{\text {det }} \cdot \rho_{a t m} \cdot \rho_{o p t} \cdot S_{\text {det }} .
$$

where $E$ is the energy emitted by the ground station, $\theta$ the divergence of the beacon, $d$ the distance between the earth and the space craft, $\lambda$ the wavelength, $\rho_{\text {det }}$ the quantum efficiency of the detector, $\rho_{a t m}$ the atmospheric transmission, $\rho_{\text {opt }}$ the transmission of the optics and $S_{\text {det }}$ the surface of the detection. With a space instrument based on a small telescope having an aperture of $100 \mathrm{~mm}$, a beacon divergence of $5^{\prime \prime}, 300 \mathrm{~mJ}$ per pulse and a distance d between $60 \times 10^{6}$ and $380 \times 10^{6} \mathrm{~km}$ (Earth-Mars distance), one obtains:

$$
1<N_{L, e}<30 \text { electrons. }
$$

The main source of noise is the solar flux reflected by the Earth. The electron flux detected by the TIPO detector is:

$$
\Phi_{\text {Sun }, e}=\frac{\lambda}{h c} \frac{S_{d e t} r_{E}^{2}}{d^{2}} \frac{\pi}{4} \cdot M_{S} \cdot A l
$$

where $M_{s}$ is the solar power received per square meter on earth in the spectral bandwidth $\delta \lambda, r_{E}$ the radius of the Earth and $A l$ the Earth albedo. The signal to noise ratio of the global detection is given by:

$$
S / N=\frac{G \cdot N_{L, e}}{\sqrt{\left(G \sqrt{G^{0.3} N_{L, e}}\right)^{2}+\frac{\delta t}{q}\left(i_{d}+\frac{4 k T}{2 \pi R q}+\frac{1}{2 \pi q}\left(\frac{u_{a}^{2}}{R^{2}}+i_{a}^{2}\right)\right)+\left(G \sqrt{G^{0.3} \Phi_{S, e} \delta t}\right)^{2}}} .
$$


where $\mathrm{G}$ is the gain of the photodetection, $\delta t$ the impulse response of the whole detection, $u_{a}$ and $i_{a}$ the voltage and current of the first detection amplifier, $\mathrm{R}$ the reading resistor of the detection and $\mathrm{T}$ its temperature. We obtain a $\mathrm{S} / \mathrm{N}$ of 1 for a distance of $10^{8} \mathrm{~km}$ which is not high enough to get a reliable detection. An active temporal filter, for instance based on a pseudo random code, is necessary to increase this $\mathrm{S} / \mathrm{N}$. A value of 30 can be obtained in the same conditions with a code having a temporal precision of 100 ns. With a compact Rubidium clock, and a dedicated miniaturised event timer, the total mass of such an instrument would be less than $15 \mathrm{~kg}$.

TIPO was first proposed in 2002 in response to the CNES call for scientific proposal for the Mars Sample Return Mission under discussion between NASA and CNES, but this mission was finally cancelled. In the meantime it was included in a technological roadmap of CNES in 2002 and in the scientific prospective of CNES in 2004. It then gave rise to a number of proposals in the framework of the ESA Cosmic Vision programme in 2005. The development of the T2L2 space instrumentation is an important step for these future interplanetary missions.

\subsection{ASTROD and ASTROD I}

The Astrodynamical Space Test of Relativity using Optical Devices ${ }^{7-10}$ (ASTROD) results from an international collaboration. The mission concept uses drag-free spacecraft in solar orbits together with a constellation of Earth orbiting satellites (including spacecraft near the Lagrange points) which provides high-precision measurement of relativistic effects, better determination of the orbits of major asteroids, improvement in the measurement of $\gamma$, measurement of solar angular momentum via Lense-Thirring effect and the detection of low-frequency gravitational waves $(50 \mu \mathrm{Hz}$ to $50 \mathrm{mHz})$. ASTROD I is planned as a single spacecraft mission and aims to perform interferometric and pulse ranging with ground stations. The scientific goals of ASTROD I include measuring the relativistic parameters with better accuracy, improving the sensitivity achieved in using the optical Doppler tracking method for detecting gravitational waves, and measuring many solar system parameters with an improvement of 1-3 orders of magnitude. In the framework of those experiments, the success of T2L2 is important to validate the concept of laser ranging with clocks and photodetection in space.

\subsection{LATOR}

According to Einstein's theory of general relativity, the Sun's gravity causes starlight to bend, shifting the apparent position of stars in the sky. The experiment Laser Astrometric Test Of Relativity ${ }^{6}$ (LATOR) led by JPL (Jet Propulsion Laboratory) would look at how the sun's gravity deflects beams of laser light emitted by two small satellites through two-way transponded laser ranging performed along each of the three sides of the light triangle and an interferometer measuring the triangle's small angle. The experience acquired with previous missions that use 
the laser ranging technique and clocks in space, such as T2L2, makes an important contribution to developing and validating such a project.

\section{Conclusion}

The T2L2 experiment will give the possibility of improving time transfer by one or two order of magnitude compared to existing microwave links like GPS and TWSTFT. Calibration of the microwave links and improvements in comparison of remote atomic clocks at a level never reached before are among the experiment's objectives. The ground experiment performed in 2004 made it possible to precisely evaluate the T2L2 accuracy and stability, which are close to the performance specifications. The launch of Jason-2 carrying the T2L2 experiment is scheduled for mid-2008. Important stakes of this mission go beyond the experiment itself: the validation of the optical time-frequency metrology in space is an important step for further missions using this kind of technology.

\section{Acknowledgments}

E. Samain and J. Weick particularly thank W.-T. Ni and the Purple Mountain Observatory for the invitation to participate and the hospitality offered during the third ASTROD Symposium held in Beijing.

\section{References}

1. P. Fridelance, E. Samain and C. Veillet,Experimental Astronomy 7 (1997) 191.

2. P. Fridelance and C. Veillet, Metrologia, 32 (1995) 27.

3. E. Samain and P. Fridelance, Metrologia 35 (1998) 151.

4. E. Samain, Applied Optics 37 (1998) 502.

5. P. Fridelance, Applied Optics 36 (1997) 5969.

6. S.G. Turyshev, M. Shao and K. Nordtvedt Jr., Class. Quant. Grav. 21 (2004) 2773.

7. W.-T. Ni, Int. J. Mod. Phys. D 11 (2002) 947.

8. W.-T. Ni, S. Shiomi and A.-C. Liao, Class. Quant. Grav., 22 (2004) 269.

9. W.-T. Ni et al., Acta Astronautica 59 (2006) 598.

10. W.-T. Ni, Int. J. Mod. Phys. D 17 (2008) 921 [arXiv: 0712.2492].

11. E. Samain, P. Bonnefond and J. Nicolas, One way laser ranging in the solar system The TIPO project on Mars, in Proc. 27th EGS General Assembly (2002).

12. E. Samain and R. Dalla, An ultra stable event timer, 13th Int. Workshop on Laser Ranging, Washington D.C., Oct. 7 - 11 (2002).

13. J. Delporte, M. Brunet and T. Tournier, Complete evaluation of a Perking Elmer RAFS in the Galileo context, in Proc. of the 14th EFTF (2000).

14. E. Samain et al., The T2L2 experiment onboard the Jason-2 space vehicle, Proc. 20th European Frequency and Time Forum - EFTF (2006).

15. P. Guillemot et al., Time Transfer by Laser Link: The T2L2 experiment on Jason-2, Proc. IEEE International Frequency Control Symposium (2006). 